\title{
ANÁLISIS DEL DISCURSO E INVESTIGACIÓN EDUCATIVA: DOS CASOS DE RESÚMENES DE CONFERENCIAS VENEZOLANOS, 1990-2002
}

\author{
Carlos Eduardo Blanco
}

\begin{abstract}
RESUMEN
Desde una perspectiva teórica mayormente interpretativa propia de los estudios sociolingüísticos y del discurso actuales, este trabajo analizó, mediante metodología micro-sociolingüística de análisis de movimientos, dos bases de datos venezolanas (273 resúmenes de ponencias de investigaciones culminadas, de los cuales se depuraron aquellos de proyectos e investigaciones en curso) presentadas en los dos más importantes eventos de la comunicación científica en educación del país (AsoVac y UCV, período 1990-2002). Como hallazgos importantes sobresalen los siguientes: el tipo más frecuente de resumen sólo evidencia la introducción, mientras que casi cuatro de cada diez no evidencian la metodología. Algo menos de la mitad de los resúmenes muestran una secuencia de cuatro o de tres de las estructuras canónicas recomendadas internacionalmente. A pesar de una leve mejoría en las estructuraciones para los dos primeros momentos del eje diacrónico de la observación (1990-1993 y 1994-1998), tal mejoría se pierde en el último momento (1998-2002). Se concluye que los hallazgos tienen implicaciones no solamente estilísticas o retóricas, sino también epistemológicas y prácticas. Se sostiene que puede ser necesaria la aplicación de principios y técnicas del análisis del discurso para mejorar la comunicación científica en este particular.

Palabras clave: investigación micro-sociolingüística, análisis del discurso, resúmenes de conferencias, investigación educacional.
\end{abstract}

\begin{abstract}
Mainly from an interpretive standpoint corresponding with current discourse studies, this paper describes and analyzes using micro-sociolinguistic movement analysis techniques, two databases of Venezuelan education conference abstracts. The corpus, consisting of 273 abstracts from the two most important research events in this country, spans approximately twelve years (1990-2002). Depuration of abstracts representing both research projects and studies in process was conducted, leaving only those referring to completed research. According to the discourse movement analysis made, the most salient findings are: the most frequent type of abstract is one that shows only the introduction (I) movement. Also, about four out of ten abstracts do not evidence the methodology (M) movement. Additionally, less than half of the abstracts are structured using either three or four of the internationally recommended structures. Despite a slight improvement in abstract structuring along the first part of the time span (1990-1993 and 1994-1998), such improvement is lost in the third moment (1998-2002). As a conclusion, it can be said that these findings may have not only linguistic implications, but also epistemological and practical ones as well. In this sense, application of principles and techniques of discourse analysis in order to improve scientific communication in this regard is recommended.
\end{abstract}

Key words: micro-sociolinguistic research, educational research, discourse analysis, conference abstracts.

M.Ed. Carlos Eduardo Blanco. Profesor Asociado, Departamento de Lengua y Comunicación, Escuela de Educación, Universidad Central de Venezuela.

Correo electrónico: ceblan@cantv.net

Recepción: 10-03-2005

Aceptación: 19-05-2005 


\title{
1. Introducción
}

\author{
La exposición científica se estructura según ciertos patrones \\ de organización retórica, la cual, con cierta tolerancia hacia \\ la variación estilística individual, impone una concordancia entre \\ los miembros de una comunidad científica, sin importar qué idioma utilicen \\ (Widdowson 1979: 61. Traducción del autor).
}

Este trabajo, el cual busca ser una aplicación de los estudios lingüísticos (microsociolinguiística) al conocimiento de la educación, ha tenido como objetivo general el obtener conocimientos específicos acerca de la estructura discursiva de investigación comunicada en el campo de la educación en Venezuela. Los objetivos específicos consisten, primero, en determinar la estructura interna de un corpus de resúmenes de dos eventos de investigación educacional; segundo, en comparar dicha estructura con parámetros comúnmente aceptados; tercero, comparar la estructura de los resúmenes en los dos eventos estudiados; y cuarto, analizar la evolución de dicha estructura en cuanto al eje diacrónico en el período estudiado. La sustentación teórica del trabajo se encuentra en las tradiciones funcionalistas e interpretativistas de la perspectiva del uso en lingüística y sociolingüística (Johnstone 2000; Butler y otros 1999; Bolívar 1998; Swales 1990) y tiene antecedentes en estudios con la variedad textual del resumen de artículos y de conferencias (Bolívar 1999, García 1999, Salager-Meyer 1992).

\subsection{Los resúmenes de investigación}

El resumen de investigación más antiguo y más estudiado es el que acompaña a los artículos de revistas especializadas, a partir de donde parece, si no haber evolucionado, por lo menos haber sido transferido hasta configurar otras sub-variedades, de manera tal que la teoría es utilizable para el análisis de otros tipos de resúmenes.

Según Teun Van Dijk (1980), los resúmenes funcionan como discursos independientes, así como indicadores previos del contenido y estructura del texto que les sigue; en los de Charles Bazerman, su entidad es la de "representación" y su esencia es la "destilación", además de que tienen un carácter que los hace "fáciles de reconocer" (Swales 1990). Fieles a lo que contemporáneamente se considera una perspectiva o postura interpretativista, los estudios de variedades textuales, de impronta linneista en su inclinación hacia las taxonomías, han solido considerarlos como "tipos ideales" que, en la práctica, pueden desviarse del ideal de diversas maneras, de tal forma que es de sumo interés indagar acerca de cuándo, por ejemplo, un resumen comienza a ser un resumen y cuándo deja de serlo, interés este de incontrovertible carácter semántico-pragmático.

Así, dentro de las variedades textuales asociadas al proceso de investigación, el resumen ha dejado sentada su importancia, sobre todo desde el punto de vista bibliotécnico, mayormente con referencia a artículos de investigación publicados.

Es de recordar que a mediados de la década de los ochenta, Naomi Graetz fundamentó la posición de que la estructura interna más común para un resumen de revista de investigación era la de P-M-R-C: Problema, Método, Resultados, Conclusiones, lo cual puede haber llevado a sostener que por lo menos la mayor parte de tales resúmenes reflejan 
el mismo patrón de los artículos a los cuales acompañan, pero con "una o dos oraciones para cada sección" (Swales 1990).

Los resúmenes, desde el punto de vista de su contenido, se organizarían entonces de la siguiente manera: a) Introducción: constituye aquella sección del resumen que contiene el problema por ser investigado, los antecedentes, los objetivos (¿qué información previa existe y qué se ha hecho?); b) Metodología: menciona los participantes en el estudio, los instrumentos utilizados y los procedimientos empleados (¿cómo se realizó el estudio?); c) Resultados: sección que presenta los datos y logros obtenidos (¿qué se encontró o evidenció?); d) Conclusiones: parte que señala las derivaciones, implicaciones, reflexiones o aplicaciones del estudio (¿cómo se evalúan los resultados?).

Es de señalar que los criterios mencionados para evaluar la estructura interna de los resúmenes se presumen razonablemente adecuados para los eventos de la comunicación investigados en el presente trabajo, pues, sin dejar de atender la especificidad del conocimiento de la educación y sin imponer un esquema rígido de las ciencias más consolidadas, ambos eventos poseen el nivel científico y la tradición suficientes dentro de la cultura occidental, de la cual es parte Venezuela. De esta manera, se cree que la estructura del resumen debería, en general, responder a la manera como se manifiesta, o se espera que se manifieste, el pensamiento científico en eventos como los estudiados.

\subsection{Los resúmenes para conferencias o congresos}

Este tipo de resúmenes se ha venido incorporando como uno de aquellos susceptibles de ser estudiados por quienes se interesan en la investigación y su discurso.

No obstante, como suele ser el caso, la palabra "resumen" no es de significado unívoco y puede denotar cuestiones diferentes en distintos contextos y para diferentes personas. Por ejemplo, no es lo mismo esa especie de reiteración que suele colocarse al final de algunos capítulos de libro o artículos de revista, que aquella versión más corta de un texto fuente para propósitos de estudio, memorización o repaso realizada por alumnos, ni lo que hoy se conoce como abstract de investigación, o representación abreviada y precisa de un trabajo o documento de investigación. En el presente trabajo se trata de ese trozo de texto corto (usualmente medido en unos pocos centenares de palabras), el cual se le solicita a los interesados de manera anticipada (por lo general unos pocos meses) a la realización de algún evento de la comunicación científica disciplinaria, es decir, conferencias, congresos o jornadas; requerimiento que usualmente se solicita con la finalidad de evaluar la posible pertinencia y calidad de la comunicación que va a ser presentada.

En este trabajo, se analizarán dos muestras de resúmenes de conferencias de educación correspondientes al ámbito venezolano, más específicamente, 273 resúmenes que, según la depuración realizada en la lectura, refieren a investigaciones culminadas, comunicaciones que se extienden por un lapso aproximado de doce años. Tales comunicaciones pueden considerarse muy representativas de lo mejor que se comunica en el contexto venezolano, habida cuenta de la tradición que representan, por una parte, el más importante evento de la comunicación científica en el país (Jornadas Anuales de la Asociación Venezolana para el Avance de la Ciencia, AsoVac, establecidas en 1950) y, por otra, la primera y más importante universidad autónoma nacional, la Central o UCV, por intermedio de su Escuela de Educación, cuyas jornadas datan de 1978. 


\section{Aspectos metodológicos}

Como categorías de análisis utilizadas dentro de los resúmenes (variedad textual objeto de estudio), se escogieron los movimientos o movidas (unidad de desarrollo del discurso capaz de encerrar los tópicos) como partes del texto que tienen una función determinada. Ello con el fin de describir el patrón de organización que presentan los resúmenes y compararlo con patrones internacionalmente recomendados, tales como los de Introducción (I), Metodología (M), Resultados (B) y Conclusiones (C): IMRC. Luego, se recolectaron los datos de lenguaje natural a partir de los resúmenes, los cuales ya habían sido informatizados desde su fuente original en papel ubicada en archivos tradicionales (no se tomaron de la internet). A partir de la lectura de tales resúmenes, la evidencia lingüística representada por los movimientos discursivos de introducción, metodología, resultados y conclusiones (la cuatríada IMRC) era copiada a mano con lápiz de grafito sobre las matrices o plantillas de recolección de datos diseñadas para tal fin, matrices cuyo prototipo había ya sido presentado a tres investigadores expertos (dos del área de sociolingüística y uno de educación), quienes emitieron una opinión en su mayor parte favorable.

Los instrumentos de recolección de la evidencia lingüística fueron concebidos por el investigador atendiendo, primero, a los objetivos del estudio, y segundo, al contenido y la estructura del texto (tópicos y actos). Los comentarios acerca de información interesante, inesperada, útil o curiosa que apareciera sobre la marcha del análisis eran anotados en los márgenes de la plantilla o matriz.

Además, se examinó un aspecto específico a la vez para no correr el riesgo de confundir información emanada del texto. Se hizo énfasis en el contenido, el orden en que se presentaban los tópicos (posible discontinuidad) y el cambio de tópico, siempre suministrando basamento gráfico a la observación. Las categorías textuales, señaladas con colores, fueron convertidas en codificaciones con sus respectivas letras y luego graficadas mediante un programa de computación.

En cuanto a la estructura interna de los resúmenes, por ejemplo, se tiene que un resumen con sus cuatro estructuras o partes canónicas completas sería uno como el siguiente (codificado como IMRC). Cuando el movimiento esperado según la cuatríada no se evidenciaba, se colocaba una "X":

\section{Motivación al logro académico en alumnos de carreras docentes}

(I) En este estudio se intentan comprobar la existencia de diferencias significativas en la motivación al logro académico entre alumnos de distintas instituciones de formación docente. Se parte de la hipótesis que de existir tales diferencias, estas son atribuibles a la extracción social del estudiante. (M) El trabajo se realizó en una muestra de 381 alumnos seleccionados aleatoriamente entre estudiantes regulares del Pedagógico de Caracas, y de las Escuelas de Educación de las Universidades Central de Venezuela, José María Vargas, Católica Andrés Bello y Metropolitana, a las cuales se les aplicó una escala de actitud elaborada ad-hoc. ®Los resultados indican la existencia de diferencias significativas por institución en los aspectos: valoración del esfuerzo, claridad en el establecimiento de metas, dedicación al estudio, valoración de la profesión, incidencia del azar y de la suerte en el éxito escolar, y valor a las dimensiones: trabajo retador y apoyo familiar y valoración de los estudios. CAun cuando en la investigación se examina un área de competencia muy definida, cual es la motivación hacia el logro académico, ella nos revela que no es del todo procedente afirmar que el venezolano promedio se caracteriza por una pobreza motivacional, ya que pueden reconocerse subdimensiones diferenciales entre los sujetos, diferencias que nos sugieren la incidencia del hábito de clase en la estructuración del perfil motivacional de los individuos.

Un resumen con tres de las estructuras (codificación IMRX) sería como el que sigue: 


\section{El rol docente-investigador y la docencia como profesión en Venezuela}

(I) El propósito central de la investigación consistió en detectar cuáles han sido los factores que han imposibilitado la aplicación del rol de investigador por los docentes de Educación Básica. Nos propusimos lograr 5 grandes objetivos, a saber:

1. Analizar teóricamente el papel que juega la investigación como elemento que garantiza la autonomía de juicio frente a terceros, en cuanto rasgo característico de las profesiones a fin de evaluar hasta qué punto la profesión docente ha cumplido con este criterio.

2. Detectar si la poca productividad en cuanto a investigación de los docentes está asociada a una representación de la ciencia y la investigación que desestimula la realización de tal actividad.

3. Detectar el nivel de autoestima profesional del docente con respecto a otras profesiones consideradas por éstos como científicas.

4. Conocer el nivel de información de los docentes acerca del rol docente- investigador previsto en la Resolución 12 del Ministerio de Educación sobre Políticas de Formación Docente.

5. Conocer los factores que obstaculizan, posibilitan o no inciden en la implantación del Rol Docente-Investigador.

(M) La metodología empleada la resumimos de la siguiente manera:

Como frase previa a la elaboración del instrumento significativo (cuestionario), se realizaron 75 entrevistas no estructuradas a docentes de Educación Básica residentes en Caracas y Barquisimeto. De allí se obtuvieron los insumos para la elaboración del Cuestionario definitivo, el cual se aplicó a una muestra de 804 docentes de Educación Básica de 5 regiones del país: Caracas, Barquisimeto, Barcelona, Ciudad Bolívar y Amazonas.

® Los resultados evidenciaron que los docentes entrevistados en su gran mayoría poseen rasgos que se acercan a una representación de la Ciencia y la Investigación asociadas al cientificismo y al experimentalismo, lo cual se traduce entre otras cosas en concebir la actividad científica como una tarea de expertos, ligada al laboratorio y a las ciencias naturales y físico-químicas. Asimismo se detectó que los niveles de información sobre el rol de investigador (Resolución 12 del M.E.) son prácticamente nulos dado que apenas un poco más del $20 \%$ demostró cierto conocimiento sobre el contenido del mismo. El docente muestra una inclinación positiva hacia la investigación, pese a considerarla, en muchos casos, ligada exclusivamente a las ciencias naturales o reducida a la actualización de conocimientos.

\section{Mientras que uno con dos estructuras (codificación IMXX) sería el siguiente:}

\section{Factores explicativos del origen de las ideas previas}

(I) Se pretende formular hipótesis que expliquen cómo se forman las ideas previas de estudiantes de Básica y Media Diversificada. Para ello se ha trabajado con el concepto de embarazo humano en estudiantes de diferentes grados y niveles del sistema educativo, ya que el aumento de la frecuencia del embarazo precoz en nuestro país se está convirtiendo en un grave problema individual y social. Se eligieron diferentes factores que pudieran incluir en la naturaleza y origen de las ideas previas.

Estos factores son:

- $\quad$ curso escolar - edad

- $\quad$ sexo

- calidad del estudiante

- origen urbano o rural de los padres

- nivel educativo terminado de la madre 
- nivel económico, medido por el tipo de vivienda

- religiosidad de familia

(M) En este estudio de casos, se siguieron los siguientes pasos, dentro de la metodología utilizada:

a) Se definieron la ideas previas de los estudiantes, por medio de la entrevista no estructurada y ayudada por dibujos.

b) Se utilizó en cuestionario de tipo de papel y lápiz, para obtener los datos correspondientes a los factores hipotéticos.

c) Se tabularon los datos con el programa SPSS.

Los resultados se presentarán en la exposición.

Por último, uno con sólo una de ellas (codificación IXXX) sería este:

El problema educativo en la oposición marxista a Juan Vicente Gómez (1926-1935)

(I) El tema que ofrecemos como ponencia tiene por objetivo central presentar las ideas que sobre la educación expusieron los grupos marxistas, tanto los del exilio, como los establecidos en Venezuela, bajo la dictadura de Juan Vicente Gómez. Nos referimos al Partido Revolucionario Venezolano (PRV), a la Agrupación Revolucionaria de Izquierda (ARDI), y al Partido Comunista de Venezuela (PCV). Esas ideas, esas preocupaciones por la educación, estarán en permanente contacto con las muy específicas condiciones económicas, sociales y políticas del país. También serán expresiones de sus particulares desarrollos ideológicos y políticos como grupos en formación doctrinaria. En el análisis de la realidad venezolana y de las acciones educativas que proponen habrá coincidencias y matices que, en algunos casos derivarán en disposiciones divergentes. La alfabetización de las mayorías, la reforma de la educación pública, las escuelas técnicas industriales y agrícolas, las universidades populares, el papel del Estado, el nuevo enfoque de los contenidos, serán los temas y problemas que ya asoman en los documentos y discusiones de esos grupos políticos. Un aspecto importante: no será una exposición del tema educativo hecha por pedagogos, por "especialistas". Todos ellos son aspirantes a revolucionarios, a políticos profesionales. El debate en firme, con filosofía y sistema lo liberarán a partir del año 36. Pero esa valorización del tema educativo que hicieron esos marxistas opuestos a Gómez, arroja nuevas luces sobre el modelo de desarrollo por ellos discutido y sobre la táctica que seguirán una vez que regresen a Venezuela a hacer realidad sus respectivos proyectos.

La clasificación que se acaba de mostrar no implica que no pueda haber o no haya otras combinaciones posibles (IXRX), (IXRC), (IMXC), etcétera. Tampoco implica que siempre se materialice un ideal de orden y continuidad, pues es usual encontrar resúmenes cuyas estructuras presenten discontinuidad (como para no contradecir la noción de discurso: ir y venir); como por ejemplo:

\section{Los aprendizajes afectivos en y para la educación básica}

(I) Los propósitos del estudio fueron ampliar conocimientos sobre los aprendizajes afectivos, determinar la situación real de estos aprendizajes en las dos primeras etapas de la Educación Básica. Así como también, establecer su contribución a la formación integral del educando y dar sugerencias para su tratamiento instruccional. (M) Para lograr los propósitos, se examinó el tratamiento dado a estos aprendizajes en la programación didáctica y en la práctica instruccional de los docentes. Se analizaron los objetivos programáticos de todas las áreas curriculares en las referidas etapas, también se revisaron, muestra de planes de trabajo de los maestros y mensajes escritos en las boletas escolares de instituciones públicas y privadas de la zona metropolitana de Caracas. (I) El análisis se realizó a partir de un referente conceptual- metodológico, con los posibles tipos de aprendizaje afectivo a ser atendidos por la escuela, y posibles estrategias didácticas para su atención. Este referente sirvió de pauta para juzgar el tratamiento dado a dichos aprendizajes y se ofrece como una propuesta pedagógica para estimular y propiciar su tratamiento instruccional. ® Los resultados encontrados indican una 
marcada desproporción en la distribución de los aprendizajes afectivos, cognoscitivos y psicomotores en los programas de ambas. Hay un alto predominio de objetivos para los conocimientos, quedando relegado el desarrollo motriz, y la relación socio-afectiva totalmente marginada. El tipo de aprendizaje afectivo más frecuente es el de los hábitos y prácticas de normas, pero sin atención instruccional apropiada. Los menos atendidos son los destinados al cultivo de sentimientos, valores, actitudes y cualidades morales escasamente se hacen presentes, y en forma esporádica, sin seguir una secuencia didáctica en se enseñanza. El desarrollo de sentimientos como el de identidad nacional y los comportamientos democráticos, no pasan de ser alusiones tangenciales. No hay un tratamiento para ayudar a formar y esclarecer valores, ni siquiera para valoración consciente del sistema democrático, ni de otros constructos declarados deseables en los principios de la Educación Básica, y en la finalidad la educación en Venezuela. Lo observado en la practica instruccional agrava la situación antes descrita, por que los maestros centran su atención en los contenidos de las asignaturas. En las boletas sus mensajes refuerzan los hábitos de estudio, y el reconocimiento a la calificación obtenida. Los aspectos formativos no se aluden, pero sí se hicieron presentes observaciones negativas, las cuales pueden afectar el desarrollo emocional y la adaptación y retención del niño en el medio escolar. (C) Los hallazgos encontrados en este estudio hacen dudar de la pregonada formación integral ofrecida en la escuela básica a nuestros educandos.

Como puede notarse, un resumen con estructura IMRC presenta discontinuidad, pues el tercer movimiento, posterior al de metodología, corresponde al de introducción, que ya se expresó en el primero (la autora del resumen "va y viene"). No obstante lo anterior, la codificación de las observaciones no registrará la posible discontinuidad, pues debe remitirse sólo a cuatro espacios, uno por movimiento, privilegiando el principio de "presencia versus ausencia", donde la ausencia es representada por una equis $(\mathrm{X})$.

\section{Análisis de resultados}

\subsection{Primera base de datos (AsoVac)}

Tabla 1. Codificación de casos observados AsoVac 1990-2002 n=144

\begin{tabular}{|c|c|}
\hline CÓDIGOS & TOTAL \\
\hline IMRX & 38 \\
\hline IXXX & 35 \\
\hline IMRC & 30 \\
\hline IMXC & 21 \\
\hline IMXX & 9 \\
\hline IXXC & 4 \\
\hline IXRX & 3 \\
\hline IXRC & 2 \\
\hline XMRC & 1 \\
\hline MIRC & 1 \\
\hline
\end{tabular}


Gráfico $\mathrm{N}^{\circ}$ 1. Frecuencia de casos observados AsoVac 1990-2002

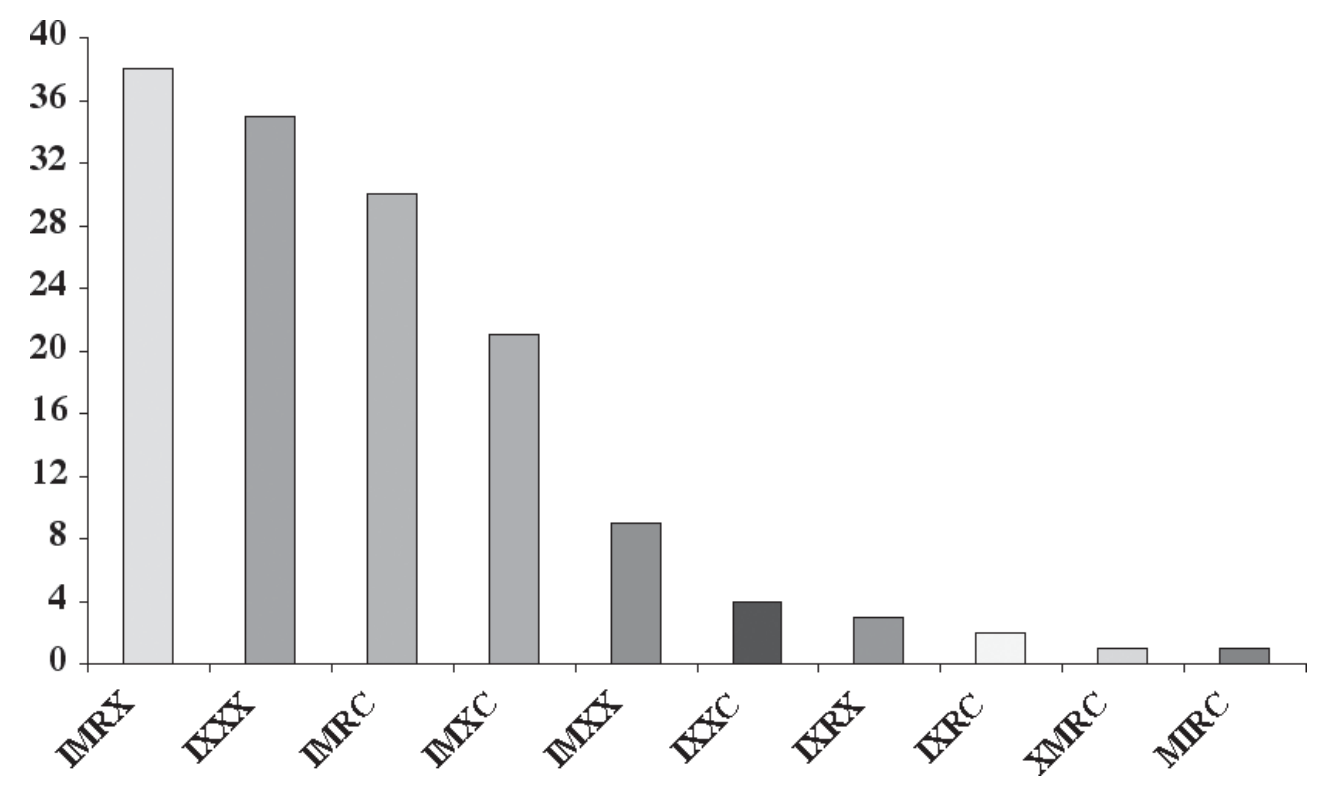

Tabla 2. Estructura interna AsoVac 1990-2002

\begin{tabular}{|l|c|}
\hline $\begin{array}{c}\text { ESTRUCTURA } \\
\text { INTERNA AsoVac } \\
\text { 1990-2002 }\end{array}$ & TOTAL \\
\hline Introducción & 143 \\
\hline Metodología & 100 \\
\hline Resultados & 75 \\
\hline Conclusiones & 59 \\
\hline
\end{tabular}


Tabla 3. Codificación de casos observados AsoVac 1990-1993

\begin{tabular}{|c|c|}
\hline CÓDIGOS & TOTAL \\
\hline IXXX & 11 \\
\hline IMXC & 9 \\
\hline IMRX & 8 \\
\hline IMRC & 8 \\
\hline IMXX & 2 \\
\hline IXXC & 2 \\
\hline IXRX & 1 \\
\hline
\end{tabular}

Tabla 4. Codificación de casos observados AsoVac 1994-1997

\begin{tabular}{|c|c|}
\hline CÓDIGOS & TOTAL \\
\hline IMRX & 18 \\
\hline IXXX & 17 \\
\hline IMRC & 8 \\
\hline IMXX & 5 \\
\hline IMXC & 2 \\
\hline IXXC & 1 \\
\hline IXRX & 1 \\
\hline IXRC & 1 \\
\hline XMRC & \\
\hline
\end{tabular}


Tabla $N^{\circ}$ 5. Codificación de casos observados AsoVac 1998-2002

\begin{tabular}{|c|c|}
\hline CÓDIGOS & TOTAL \\
\hline IMRC & 14 \\
\hline IMRX & 12 \\
\hline IMXC & 8 \\
\hline IXXX & 7 \\
\hline IMXX & 2 \\
\hline IXRX & 1 \\
\hline IXRC & 1 \\
\hline MIRC & 1 \\
\hline
\end{tabular}

Desde el punto de vista de un análisis del uso de las cuatro estructuras canónicas a lo largo del eje diacrónico, se tiene que para el primer momento de la observación (1990-1993) los resúmenes que presentan sólo introducción (IXXX) tienen la frecuencia mayor (11). Aquellos que presentan tres o cuatro estructuras (IMRX-IMRC) constituyen algo más de la tercera parte del total, mientras que aproximadamente dos de cada tres evidencian la metodología. La estructura más utilizada es la de introducción con 41 (la totalidad), mientras que la menos utilizada es la de resultados con diecisiete (17).

Para el segundo momento (1994-1997), la frecuencia mayor, con 18 casos, la representan aquellos resúmenes casi completos, a los cuales les faltan solo las conclusiones (IMRX). Casi parejo con el primero, el segundo tipo de caso más frecuente es el de los resúmenes con sólo la introducción, con 17 casos, casi uno de cada tres. Los resúmenes con sus cuatro partes completas (IMRC) son ocho, menos de dos de cada diez. Los que evidencian tres o cuatro estructuras son 26, poco menos de la mitad. Aproximadamente dos de cada tres muestran la metodología. La estructura más utilizada es la de introducción con 56 casos (todos excepto uno) y la menos utilizada la de conclusiones con dieciséis (16).

Para el tercer momento (1998-2002), en el cual ocurre una disminución en la cantidad de comunicaciones, el tipo de resumen más frecuente en la base de datos de la AsoVac es el que tiene todas sus partes completas, con catorce casos, aproximadamente uno de cada tres. El segundo más frecuente es aquel al que solo le faltan las conclusiones, con 12, poco menos de uno de cada cuatro, por lo que puede decirse que algo más de la mitad evidencia tres o bien cuatro estructuras. En cuanto a la evolución en la evidenciación de la metodología en este período final, 37 resúmenes lo hacen, cuatro de cada cinco. La estructura más usual es la de introducción con 45 casos (todos excepto uno); y la menos, la de conclusiones con 24 (casi la mitad de los casos no las evidencian).

Así, al comienzo de la observación (1990-1993), aproximadamente dos de cada tres resúmenes evidenciaban la metodología, mientras que al final de ésta (1998-2002), lo hacían cuatro de cada cinco, una mejora digna de reconocer. Por último, el examen a lo largo del eje diacrónico hace posible afirmar que para el caso de la AsoVac es perceptible una leve mejoría en la calidad de la estructuración de los resúmenes, esto sugerido particularmente por el mayor uso gradual de tres o cuatro estructuras (IMRX o IMRC) desde el inicio del resumen, aunque no se puede dejar de notar que la frecuencia en el uso de las cuatro estructuras canónicas completas puede considerarse baja (ver tablas 3, 4 y 5). 


\subsection{Segunda base de datos. UDI-UCV (1990-2000)}

Del total de casos, se obtuvo que la mayor frecuencia, con 50, correspondió a la estructura IXXX, es decir, aquella que sólo manifiesta la introducción. La segunda estructuración más frecuente, con 42 casos, fue la de IMRC, es decir, el resumen que presenta completas sus cuatro partes canónicas. La tercera estructuración más frecuente fue la de IMRX, aquella que no manifiesta las conclusiones. Es de señalar que 57 de los 129 casos posibles, es decir, menos de la mitad, manifiesta tres o cuatro estructuras, y que 73, bastante más de la mitad, no evidencian la metodología. La estructura más frecuentemente utilizada es la de introducción, con todos los casos; y la menos utilizada, la de conclusiones con 57 casos, algo menos de la mitad de todos los resúmenes (ver tablas 6 y 7; gráfico 2).

Tabla $N^{\circ} 6$. Codificación de casos observados UDI 1990-2000 $\mathrm{n}=\mathbf{1 2 9}$

\begin{tabular}{|c|c|}
\hline CÓDIGOS & TOTAL \\
\hline IXXX & 50 \\
\hline IMRC & 42 \\
\hline IMRX & 15 \\
\hline IMXC & 5 \\
\hline IXXC & 5 \\
\hline IXRC & 5 \\
\hline IMXX & 4 \\
\hline IXRX & 3 \\
\hline
\end{tabular}

Gráfico $\mathrm{N}^{\circ}$ 2. Codificación de casos observados UDI 1990-2000

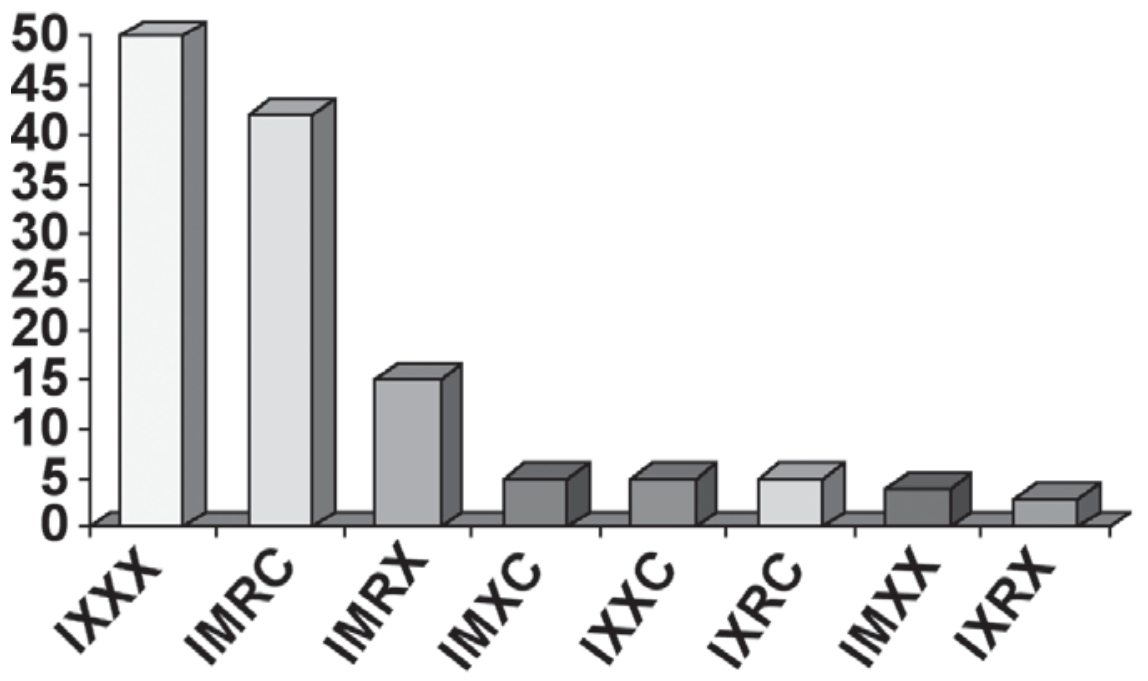


Tabla 7. Estructura interna UDI 1990-2000

\begin{tabular}{|l|c|}
\hline $\begin{array}{c}\text { ESTRUCTURA INTERNA } \\
\text { UDI 1990-2000 }\end{array}$ & TOTAL \\
\hline Introducción & 129 \\
\hline Metodología & 66 \\
\hline Resultados & 65 \\
\hline Conclusiones & 57 \\
\hline
\end{tabular}

Tabla $N^{\circ}$ 8. Codificación de casos observados UDI 1990-1993

\begin{tabular}{|c|c|}
\hline CÓDIGOS & TOTAL \\
\hline IMRC & 10 \\
\hline IXXX & 10 \\
\hline IMRX & 6 \\
\hline IXXC & 3 \\
\hline IMXX & 2 \\
\hline IMXC & 1 \\
\hline IXRX & 1 \\
\hline
\end{tabular}

Tabla $N^{\circ}$ 9. Codificación de casos observados UDI 1995-1998

\begin{tabular}{|c|c|}
\hline CÓDIGOS & TOTAL \\
\hline IMRC & 21 \\
\hline IXXX & 10 \\
\hline IMRX & 6 \\
\hline IMXC & 2 \\
\hline IXRC & 2 \\
\hline IMXX & 1 \\
\hline IXRX & 1 \\
\hline IXXC & 1 \\
\hline
\end{tabular}


Tabla $N^{\circ}$ 10. Codificación de casos observados UDI 2000

\begin{tabular}{|c|c|}
\hline CÓDIGOS & TOTAL \\
\hline IXXX & 30 \\
\hline IMRC & 11 \\
\hline IMRX & 3 \\
\hline IXRC & 3 \\
\hline IMXC & 2 \\
\hline IMXX & 1 \\
\hline IXRX & 1 \\
\hline IXXC & 1 \\
\hline
\end{tabular}

Las tablas 8, 9, y 10 indican la evolución en el uso de las estructuras de los resúmenes de la UDI en cuanto al eje diacrónico. Para el primer momento (1990-1993), predominan las estructuras IMRC e IXXX, constituyendo estas poco más de la mitad de los resúmenes. Aproximadamente la mitad de los resúmenes (16) evidencia tres o cuatro estructuras desde el inicio de la escritura del resumen, mientras que algo más de la mitad (19) evidencia la metodología. La estructura más utilizada es la de introducción, con la totalidad de los casos (33), y la menos utilizada, la de conclusiones (14 casos).

Para el segundo momento (1995-1998), el tipo de resumen más utilizado es el IMRC, es decir, aquel con sus cuatro partes completas, con 21 casos (casi la mitad), mientras que algo menos de dos de cada tres resúmenes evidencian tres o cuatro estructuras desde su inicio. Poco más de dos de cada tres resúmenes (30 casos) evidencian la metodología. La estructura más usual es la de introducción, presente en todos los casos, y la menos, la de conclusiones, presente en 26.

Para el tercer momento (año 2000), el tipo de resumen usual es el que más se aleja del tipo ideal, es decir, aquel que sólo evidencia la introducción (IXXX), con 30 casos. Catorce ítemes presentan entre tres y cuatro estructuras desde el inicio de la escritura del resumen, lo cual significa aproximadamente uno de cada cuatro. Diecisiete (17) casos evidencian la metodología, lo cual implica uno de cada tres. La estructura más utilizada dentro de los resúmenes es la de introducción, con la totalidad de los casos, mientras que las menos utilizadas son la de metodología y la de conclusiones, con 17.

Entonces, al comienzo de la observación (1990-1993), diecinueve de treinta y tres casos, poco más de la mitad de las comunicaciones, evidenciaban la metodología, mientras que al final (2000) lo hacen diecisiete casos de cincuenta y dos, es decir, aproximadamente uno de cada tres. El examen a lo largo del eje diacrónico permite afirmar que se percibe una desmejora en cuanto a la calidad de la estructuración de los resúmenes para este evento en particular; ello tomando como indicador fundamental la cantidad de resúmenes que evidencian tres o cuatro estructuras desde el inicio de su redacción. Nótese que para el comienzo del período (1990-1993), aproximadamente la mitad de las comunicaciones presentaban esta estructuración general, IMRX o IMRC, lo cual se incrementó a casi dos de cada tres para el segundo momento (1995-1998), aunque luego disminuyó a aproximadamente una de cada cuatro, reconociendo que el número de comunicaciones siempre fue en aumento. 


\subsection{Casos combinados UDI-AsoVac (1990-2002)}

En cuanto a la integración de ambas bases de datos, se obtiene que en los 273 casos estudiados, el tipo de resumen más frecuente, con 85 casos, es aquel que sólo evidencia la introducción, mientras que el segundo más frecuente, con 72 casos, es aquel que presenta sus cuatro partes canónicas completas. Los resúmenes que evidencian tres o cuatro estructuras desde el comienzo de la redacción son 125, lo cual implica menos de la mitad. En cuanto a la evidenciación de la metodología, 166 casos la presentan, es decir, aproximadamente seis de cada diez, lo que deja como derivación lógica el que aproximadamente cuatro de cada diez no la evidencian. En cuanto a resúmenes que muestren sus cuatro partes canónicas, se consiguieron 72 , poco más de uno de cada cuatro, lo cual puede considerarse bajo, sobre todo si nos referimos a investigaciones culminadas.

Además, se percibe como un hallazgo importante la evidenciación de dos grandes tendencias antitéticas entre sí: una en dirección a configurar el resumen de tipo ideal IMRC y otra que se expresa en dirección contraria, hacia la configuración del resumen de tipo "solo introducción” o IXXX, suerte de fuerzas centrípeta y centrífuga respecto del centro del método científico. Una tercera tendencia del resumen es la que excluye la estructura retórica de conclusiones.

Desde un punto de vista diacrónico, para el primer momento de la observación (19901993), prevalecen los dos tipos de resúmenes antitéticos, el IXXX, con 21 casos, y el IMRC, con 18. Algo menos de la mitad (32) presentan tres o cuatro estructuras desde su inicio, y casi dos de cada tres (46) evidencian la metodología. Para el segundo momento de la observación (1994-1998), de nuevo prevalecen los dos tipos antitéticos, el IMRC con 29 casos y el IXXX con 27. Algo más de la mitad (53 casos) presenta entre tres y cuatro estructuras desde el inicio, mientras que 66 (alrededor de dos de cada tres) evidencian la metodología, por lo cual podría afirmarse que ocurrió una leve mejoría en el segundo lapso. Para el tercer momento (19982000), la polarización entre los dos tipos antitéticos (IXXX-IMRC) se mantiene, el primero con 37 casos y el segundo con 28. Aproximadamente dos de cada cinco resúmenes se estructuran con tres o cuatro de sus partes canónicas desde el inicio, mientras que poco más de la mitad (54 casos) evidencian la metodología. Adicionalmente, casi uno de cada tres resúmenes del total (85 casos) evidencian sólo la introducción (ver tablas 11, 12, 13, 14, y gráfico 3).

Tabla $N^{\circ} 11$. Codificación de casos observados AsoVac-UDI 1990-2002

\begin{tabular}{|c|c|c|c|}
\hline CÓDIGOS & AsoVac & UDI & TOTAL \\
\hline IXXX & 35 & 50 & 85 \\
\hline IMRC & 30 & 42 & 72 \\
\hline IMRX & 38 & 15 & 53 \\
\hline IMXC & 21 & 5 & 26 \\
\hline IMXX & 9 & 4 & 13 \\
\hline IXXC & 4 & 5 & 9 \\
\hline IXRC & 2 & 5 & 7 \\
\hline IXRX & 3 & 3 & 6 \\
\hline XMRC & 1 & 0 & 1 \\
\hline MIRC & 1 & 0 & 1 \\
\hline
\end{tabular}


Gráfico No. 3. Frecuencia de casos observados AsoVac-UDI 1990-2002

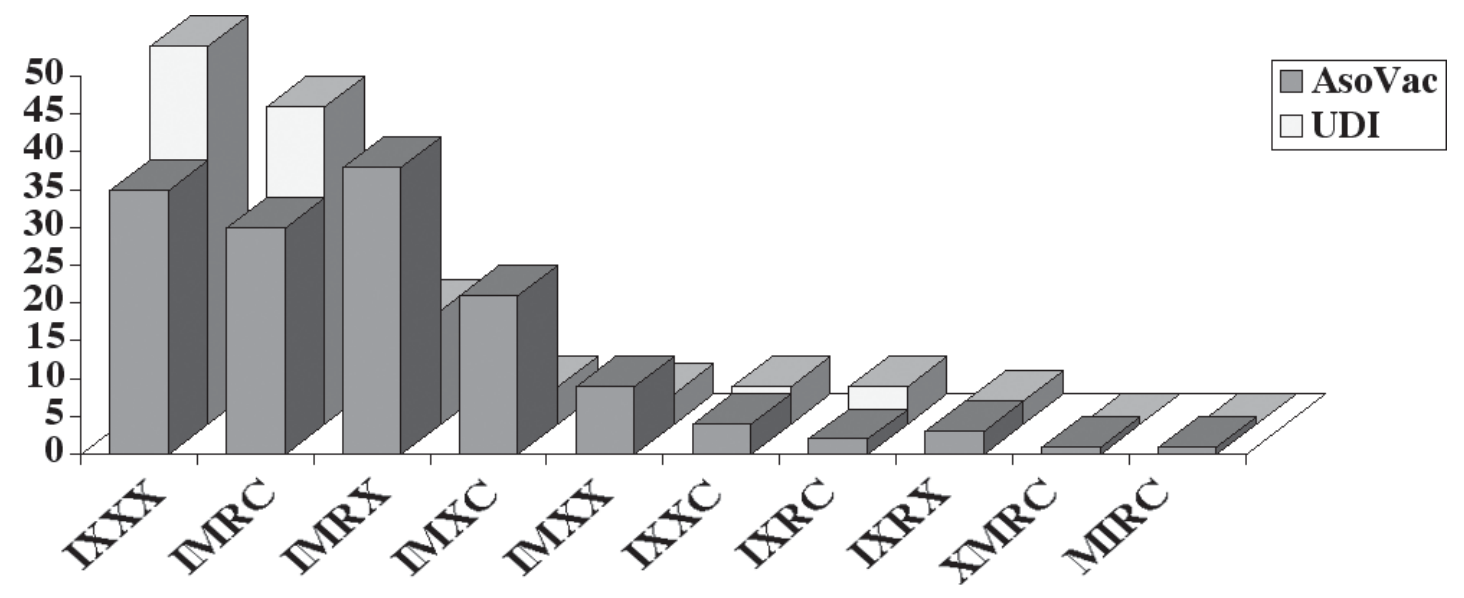

Tabla N 12. Codificación de casos observados AsoVac-UDI 1990-1993

\begin{tabular}{|c|c|c|c|}
\hline CÓDIGOS & AsoVac & UDI & TOTAL \\
\hline IXXX & 11 & 10 & 21 \\
\hline IMRC & 8 & 10 & 18 \\
\hline IMRX & 8 & 6 & 14 \\
\hline IXXC & 2 & 3 & 5 \\
\hline IMXX & 2 & 2 & 4 \\
\hline IXRX & 1 & 1 & 2 \\
\hline
\end{tabular}

Tabla N 13. Codificación de casos observados AsoVac-UDI 1994-1998

\begin{tabular}{|c|c|c|c|}
\hline CóDIGOS & AsoVac & UDI & TOTAL \\
\hline IMRC & 8 & 21 & 29 \\
\hline IXXX & 17 & 10 & 27 \\
\hline IMRX & 18 & 6 & 24 \\
\hline IMXC & 4 & 2 & 6 \\
\hline IMXX & 5 & 1 & 6 \\
\hline IXXC & 2 & 1 & 3 \\
\hline IXRC & 1 & 2 & 3 \\
\hline IXRX & 1 & 1 & 2 \\
\hline XMRC & 1 & 0 & 1 \\
\hline
\end{tabular}


Tabla Nº 14. Codificación de casos observados AsoVac-UDI 1998-2002

\begin{tabular}{|c|c|c|c|}
\hline CóDIGOS & AsoVac & UDI & TOTAL \\
\hline IXXX & 7 & 30 & 37 \\
\hline IMRC & 14 & 11 & 25 \\
\hline IMRX & 12 & 3 & 15 \\
\hline IMXC & 8 & 2 & 10 \\
\hline IXRC & 1 & 3 & 4 \\
\hline IMXX & 2 & 1 & 3 \\
\hline MIRC & 1 & 0 & 1 \\
\hline IXXC & 0 & 1 & 1 \\
\hline
\end{tabular}

\section{Conclusiones y recomendaciones}

1. A la vista de los datos analizados, puede decirse que para la etapa presente no es perceptible algún grado de progreso en la calidad de la estructuración de los resúmenes de educación para los dos casos estudiados, representativos de lo mejor que se comunica en la investigación educativa venezolana. Además, se presentaron inconvenientes debido a lo poco directo y claro que se suele ser en algunos resúmenes, por lo que debe considerarse estrictamente necesaria la explicitación de todas las secciones del resumen, muy especialmente la de metodología, pues ella forma parte del componente de objetividad de la tarea de investigar.

2. En relación con lo anterior, vale la pena señalar que la sección del resumen más fácil de precisar resultó la de introducción, y la más difícil, la de metodología, seguida de la de resultados-conclusiones. Posiblemente esto tenga que ver con que la metodología es la parte más difícil de explicitar debido al relativamente bajo entrenamiento metodológico que tiene el autor promedio.

3. También relacionado con el aspecto crítico de la metodología, en numerosos casos la denominada "metodología" no lo era de investigación, sino de didáctica. Este es un tema de importancia pues tiene que ver con la definición de investigación que se pueda estar asumiendo en la comunidad discursiva en particular. La investigación didáctica es una forma de investigación, pero parece confundirse a veces con otros géneros tales como descripciones de la didáctica instrumentada o la mera descripción de actividades de aula.

4. Desde el punto de vista de los estudios de la retórica y el discurso, el planteamiento anterior puede implicar que algunos autores de resúmenes para ponencias confundan el evento de habla "clase" con el de "informe de investigación"; de allí que exista una importante cantidad de comunicaciones que sólo evidencian la introducción (IXXX), es decir, son una exposición de información conocida: una clase común y corriente. 
5. En relación con el punto anterior, un énfasis en la "información conocida" puede tener implicaciones no sólo para el progreso del conocimiento sino para el mantenimiento, aumento o disminución de la clientela que sigue y asiste a este tipo de eventos que hemos estudiado. Centrarnos en lo conocido sin aportar información importante, por lo novedosa o controversial, podría crear tedio y hasta alejamiento de la clientela, lo cual puede incidir negativamente en el futuro de nuestros eventos de investigación. Por el contrario, si el énfasis es en la información nueva, mayormente en la forma de resultados bien sustentados por una sana metodología, ello puede no sólo mantener sino incentivar y aumentar la clientela a la que se supone que nos debemos. El hecho de que el movimiento discursivo que expresa la información nueva sea el de resultados ${ }^{\circledR}$, y que este se encuentre presente en sólo 45 de los 273 casos posibles -aproximadamente en uno de cada seis- es preocupante.

6. Otro punto importante es aquel relacionado con la extensión y estructuración de los resúmenes, con su legibilidad y evaluabilidad. El alargamiento excesivo de un resumen, a veces sin necesidad o razón aparente, tendrá su influencia sobre la estructuración del texto. Esta anomalía es notable para la segunda base de datos (UDI-UCV), con casi el doble de los parámetros internacionales. Mientras más extenso sea un resumen, más probable es que contenga información innecesaria, divagadora y distractora. Esto afectará la legibilidad y evaluabilidad de los resúmenes y, si no son rechazados, ello a su vez afectará la calidad de los que se publiquen. No obstante, este caso de "palabradicción" presenta aristas contradictorias, dialécticas, pues a veces parece que decimos demasiado cuando no debemos y muy poco cuando sí es necesario, por lo que se puede afirmar que hay "palabradicción" mezclada con "no decir lo que hay que decir". Esto puede ejemplificarse, en cuanto al decir mucho, con los resúmenes que sólo evidencian la introducción y que divagan con verbosidad. En cuanto al "decir muy poco", se consiguieron casos en los cuales, por ejemplo, la metodología era apenas mencionada, con excesiva economía de palabras, cuando esta sección del resumen es de lo más fundamental (¿para qué ahorrar en lo más importante?).

7. Hay un aspecto crítico de los hallazgos, el cual se relacionó directamente con la metodología del trabajo ya realizado, por una parte, y con el tema de la cantidad de investigaciones que se dice que existen en educación. A pesar de que se depuró el corpus en cuanto a resúmenes de proyectos e investigaciones en curso, al final aparece un número considerable de casos que sólo evidencian la introducción, además de los que no presentan resultados. La decisión acerca de la depuración se tomó debido a la intención de lograr un acercamiento lo más preciso posible a lo que podría ser la investigación realmente existente y comunicada en resúmenes. Ahora bien, si desde un punto de vista lógico, la estructura del resumen-proyecto debería ser la de IMXX y la del resumeninvestigación en curso IMRX, ¿cómo quedamos desde este mismo punto de vista con la numerosa cantidad de resúmenes de tipo IXXX? En sana lógica, deberíamos restar también todos estos últimos resúmenes para acercarnos más a la buscada investigación "realmente existente". Al realizar dicha resta, se pondría en tela de juicio la creencia de que la gran cantidad de comunicaciones recibidas en las jornadas evidencia que "ha aumentado la investigación". 
Las recomendaciones podrían resumirse en promover la producción, remisión y aprobación de resúmenes IMRC y desincentivar los IXXX. Vale la pena señalar que el estudio realizado se liga con temas no solo lingüísticos o retóricos (¿cómo escriben nuestros profesores?), sino también epistemológicos (¿dónde y cómo está la investigación?) o prácticos (¿cuánto nos ahorraríamos rechazando los resúmenes IXXX?).

\section{Fuentes documentales utilizadas}

Asociación Venezolana para el Avance de la Ciencia (AsoVac). Acta Científica Venezolana. Volúmenes 41 al 52 (Años 1990-2002). Área de Educación.

Universidad Central de Venezuela. Unidad de Investigaciones de la Escuela de Educación. Material mimeografiado (años 1990, 1991, 1993). Folletos de resúmenes (años 1995, 1996, 1998). Archivo electrónico contentivo de los resúmenes de ponencias (año 2000).

\section{Fuentes bibliográficas}

Berkenkotter, C. y T. Huckin. 1995. Genre knowledge in interdisciplinary communication. Language, cognition and power. Hillsdale, NY: Lawrence Earlbaum.

Bolívar, A. 1998. Discurso e interacción en el texto escrito. Caracas: Universidad Central de Venezuela.

1999. "Los resúmenes para eventos científicos en Lingüística Aplicada en América Latina: estructura e interacción". Opción. 15 (29): 61-81.

Butler, C.; Ricardo Mairal; Javier Martín Arista y Francisco J. Ruiz de Mendoza (Comps.). 1999. Nuevas Perspectivas en Gramática Funcional. Barcelona: Ariel.

García, J. 1999. "Un estudio comparativo de los abstracts para eventos científicos en inglés y español”. D.E.L.T.A. 15 (2): 269-288.

Johnstone, B. 2000. Qualitative Methods in Sociolinguistics. New York: Oxford University Press.

Salager-Meyer, F. 1992. "A text type and move analysis study of verb tense and modality distribution in medical English abstracts”. English for Specific Purposes. 11 (2): 107-124. 
Swales. J. 1990. Genre Analysis. Cambridge, GB: Cambridge University Press.

Van Dijk, T. (Ed.). 1980. Macrostuctures: An Interdisciplinary Study of Global Structures in Discourse, Interaction, and Cognition. Hillsdale, New Jersey: Erlbaum.

Widdowson, H.G. 1979. Explorations in Applied Linguistics. Oxford, GB: Oxford University Press. 